\title{
Niveau sozialrechtlicher Existenzsicherung und Mindestlohn in Deutschland
}

Viel öffentliche Beachtung finden in der letzten Zeit all jene Vorschläge, die einen existenzsichernden Mindestlohn in Deutschland einführen wollen. Mit Bezug auf das Niveau der Fürsorgeleistungen (Arbeitslosengeld II oder Sozialhilfe) wird ein Lohn von bis zu $750 €$ netto für einen Alleinstehenden vorgeschlagen. Verkannt wird dabei allerdings, dass das Existenzminimum eines Erwerbstätigen höher ist als das eines Nichterwerbstätigen. Ein den aktuellen Lebenshaltungskosten angepasster existenzsichernder Mindestlohn müsste Ende 2006 mindestens $945 €$ netto - oder, wenn man einen wirklich fairen Lohnstandard schaffen will, $1015 €$ netto - betragen. Welchen Beitrag können die einzelnen bedarfsorientierten Elemente der bestehenden Fürsorgeleistung zur Ermittlung dieses Mindestniveaus leisten?

\section{Von der Sozialhilfe zur Grundsicherung für Arbeitsuchende}

Nach Bder Hartz- Reform hat das neue Sozialhilferecht (SGB XII) den Charakter als „letztes Netz" einer fürsorgerechtlichen Existenzsicherung verloren. An dessen Stelle ist das SGB II (Grundsicherung für Arbeitsuchende) mit seinen vergleichbaren Leistungen zur Sicherung des Lebensunterhalts getreten. Dieses neue Netz sozialer Existenzsicherung ist allerdings mit einer Reihe noch nicht erschlossener Risiken behaftet. Es bricht mit einer Tradition, in der die Sozialhilfe in Deutschland stand, und enthält neue Sollbruchstellen: Sowohl im SGB XII als auch noch mehr im SGB II ist von bedarfsdeckenden, individuellen $\mathrm{Zu}$ satzleistungen Abstand genommen worden. Die Zusammenstellung der nun umfassenderen Pauschalsätze lässt auch die Sorgfalt bei der Ermittlung bedarfsrelevanter Bezugsdaten vermissen, wie sie bei dem vergleichbaren, 1990 eingeführten Statistikmodell noch angewandt wurde.

In dieser Umbruchsituation besteht die Gefahr, dass aus Erfahrungen bekannte Orientierungspunkte für die Bestimmung eines Existenzminimums verloren gehen. Die Frage nach der Höhe des Existenzminimums - ob im Arbeitsleben oder außerhalb - fällt so der Beliebigkeit anheim.

Spezifisch für Nachkriegsdeutschland war das 1962 geschaffene Bundessozialhilfegesetz (BSHG). Es baute auf dem grundgesetzlich garantierten Schutz der Menschenwürde auf und verkörperte diesen
Anspruch auch in vielerlei Hinsicht. Im über 40-jährigen Praxistest konnte man die Probleme gut studieren, die der Umgang mit einem solchen steuerfinanzierten, bedürftigkeitsgeprüften staatlichen sozialen Sicherungsnetz macht. Bernd Schulte und Peter Trenk-Hinterberger (1986) haben in ihrem Lehrbuch die Geschichte, die Elemente und die Komplexität dieses Systems auf einem seitdem nicht mehr erreichten Niveau zusammengestellt.

Dass es auch viele Sperren und Filter bei der Inanspruchnahme des BSHG gab, das hat schon Stefan Leibfried (1976) früh aufgezeigt. So wurde der Anspruch auf Existenzsicherung im rechtlichen Dunstkreis kommunaler Leistungsverwaltung gelegentlich durchlöchert. Aber der Anspruch, ein ,letztes Netz" bedarfsdeckender Sicherung als Voraussetzung für ein menschenwürdiges Leben zu spannen, bestand bis zur Hartz- Reform. Leichtfertige Sozialwissenschaftler haben dieses Bild vom „letzten Netz" im Rahmen der Aktivierungsdiskussion der 1990er Jahre abgewertet und lächerlich gemacht, ließen es zum Bild der wohlfahrtsstaatlichen „Hängematte" mutieren, um die Umformung zum "Trampolin"des aktivierenden Sozialstaats zu begründen.

\section{Elemente des sozialhilferechtlichen Existenzminimums}

Um die grundlegenden Veränderungen zu verdeutlichen, wird zunächst das , alte “ System der Sozialhilfe betrachtet und disku- tiert, welcher Erkenntnisgewinn daraus für die aktuelle Mindestlohndiskussion gezogen werden kann. Bedarfselemente des sozialrechtlichen Existenzminimums der Sozialhilfe waren Regelsätze sowie Mehrbedarfe und Unterkunftskosten. Sie mussten in jeweils aufwendigen Verfahren ermittelt werden.

\subsection{REGELSÄTZE}

Der Bereich der Armenfürsorge wurde mit dem BSHG wissenschaftlich durchdrungen und entwickelt. Sehr deutlich wird dies anhand des Verfahrens zur Ermittlung des Niveaus der Regelsätze. Das soziokulturelle Existenzminimum wurde gutachterlich ermittelt: Zunächst wurde ein Warenkorb für den notwendigen Lebensunterhalt mit den dazugehörigen Preisen zusammengestellt und daraus die Höhe der Regelsätze nachprüfbar abgeleitet (Warenkorbmodell). Auch wenn manches lächerlich erschien die vielen Liter Kondensmilch, die „halbe“ Kinokarte, die 2 Rollen Klopapier - es hatte den Charme der Anschaulichkeit und der Transparenz. Die Armseligkeit des beim Deutschen Fürsorgetag 1980 auf dem Tisch präsentierten Monatswarenkorbs ei-

Helga Spindler, Dr., Professorin für Öffentliches Recht, Sozialrecht und Arbeitsrecht. Universität Duisburg-Essen, Fachbereich Bildungswissenschaften, Institut für Soziale Arbeit. Arbeitsschwerpunkte: Sozialhilfe, Grundsicherung für Arbeitsuchende, Beschäftigungsförderung, aktivierender Sozialstaat, prekäre Arbeitsverhältnisse. e-mail: helga.spindler@uni-duisburgessen.de 
nes Sozialhilfeempfängers stieß eine kritische Debatte an, die schließlich zum Alternativwarenkorb von 1985 führte. ${ }^{1}$

Die Koordination dieser Aktivitäten und vieler weiterer fachlicher Empfehlungen zum Verwaltungshandeln der Sozialhilfe lag lange Zeit beim „Deutschen Verein für öffentliche und Private Fürsorge" (DV), der in seinen Arbeitsgruppen Wissenschaftler und Fachleute von Behörden und Verbänden zusammenführte. Der Bund setzte den gesetzlichen Rahmen mit der Regelsatzverordnung. Diese unterlag der $\mathrm{Zu}-$ stimmung der Länder im Bundesrat. Die Finanzierung des Existenzminimums war Sache der Kommunen. Das machten sie zwar auch nach der Einführung des Rechtsanspruchs auf Sozialhilfe nicht gerne. Dennoch waren sie lange Zeit auch wegen der moderierenden Rolle des Deutschen Vereins bereit, das Verfahren der Bedarfsermittlung zu akzeptieren und sich an den erarbeiteten Empfehlungen zu orientieren.

Im Jahr 1990 wurde ein Statistikmodell eingeführt. Nunmehr wurde neben der Entwicklung der Nettoeinkommen und der Lebenshaltungskosten das aus der vergangenen Einkommens- und Verbrauchsstatistik (EVS) ablesbare Konsumverhalten unterer Einkommensgruppen zu einer wichtigen Bezugsgröße für die Regelsätze. Dieser Statistikregelsatz war - auch wenn er zunächst noch zu einer strukturellen Erhöhung der Leistung in den Jahren 1990 bis 1992 von zusammen etwa 9 \% führte - wesentlich abstrakter und damit manipulationsanfälliger. So konnten die Regelsätze der Haushaltsanghörigen, für die die Warenkörbe vorher noch getrennt gepackt worden waren, mit der angewandten Differenzmethode nicht mehr nachvollziehbar bestimmt werden. Auch die Wahl der statistischen Vergleichsgruppen, die mit ihrem Einkommen ja verlässlich über der Sozialhilfeschwelle liegen mussten, blieb strittig, was nicht zuletzt den Datenlücken der EVS geschuldet war. Die Hochrechnungen der einschlägigen Lebenshaltungskosten waren gleichfalls nie ganz überzeugend. ${ }^{2}$

Trotz der mittlerweile vorhandenen Spargesetzgebung stand es bis 2003 nicht zur Diskussion, dass das Existenzminimum jedes Jahr angepasst werden sollte, um dem Grundsatz der Bedarfsdeckung zeitnah zu entsprechen. Die Akzeptanz, die dieses System der auf die Lebenshaltungskosten bezogenen Bedarfsermittlung gewonnen hatte, wurde besonders deutlich, als das Bundesverfassungsgericht in seiner Entschei- dung über das steuerliche Existenzminimum auf diesen Maßstab zurückgegriffen hat. Politisch umstritten war lediglich die Höhe der Anpassung.

Seit 2003 ist dieser Regelsatz praktisch eingefroren, obwohl erhebliche Gesundheitskosten mit der Gesundheitsreform 2004 neu hinzukamen, Preissteigerungen in allen Bereichen, besonders bei Energie und Nahverkehr, zu verzeichnen sind und Freibeträge für sozial Schwache, wie z. B. bei Rundfunk- und Fernsehgebühren, weggefallen sind.

Gleichwohl sieht auch die neu gefasste Regelsatzverordnung ein der früheren Sozialhilfe entsprechendes Verfahren zur Ermittlung des Existenzminimums vor: Statt der Expertengruppen beim Deutschen Verein werden nun zentrale Gutachten vom jeweils zuständigen Bundesministerium in Auftrag gegeben. Die Ergebnisse dieser Expertisen sind allerdings schon der Fachöffentlichkeit kaum mehr zugänglich. Im Gegensatz zur früheren Regelsatzfestlegung wird auch keine gesellschaftsweite sozialpolitische Diskussion mehr geführt, und dies, obwohl der neue Eckregelsatz von $345 €$ Vielen als unzureichend gilt. Der Paritätische Wohlfahrtsverband (DPWV) hat deshalb eine umfangreich begründete Forderung aufgestellt, den Regelsatz aktuell auf $415 €$ zu erhöhen, um den gesetzlichen Vorgaben zu genügen (DPWV 2006, S. 15; Martens 2006).

Dass die Politik dieser Forderung folgt, darf bezweifelt werden: Vor allem durch die institutionellen Veränderungen ist eine neue Dynamik entstanden, die das Niveau der Leistung gefährdet. Seit der Bund anstatt der Kommunen für die Finanzierung dieser Regelleistungen nach dem SGB II zuständig ist, ist sein ökonomisches Eigeninteresse gewachsen, diese Leistungen möglichst niedrig zu halten. Die moderierende Rolle des Deutschen Vereins wird von ihm nicht mehr angefragt.

\subsection{MEHRBEDARFE UND EINMALIGE BEIHILFEN}

Zusätzlich zum Regelsatz gab und gibt es auch noch pauschalierte Mehrbedarfe, die auf bestimmte, bedarfsaufwendigere Lebenslagen Bezug nehmen: z. B. Mobilitätsbeschränkung, kostenaufwendige Diäten, Schwangerschaft, Alleinerziehen, Teilnahme an Maßnahmen der beruflichen Rehabilitation, ursprünglich auch Rentenalter. Allerdings sind damit keinesfalls alle auf- wendigeren Lebenslagen abschließend erfasst, vor allem nicht die intensive Arbeitssuche oder Weiterbildung und seit 1993 auch nicht mehr die Erwerbstätigkeit mit Einkommen unterhalb der Sozialhilfeschwelle (siehe Abschnitt 4). Unklar blieben auch immer die statistischen Bezugsgrößen für die Mehrbedarfe, obwohl der Deutsche Verein ursprünglich auch dafür eine Art Warenkorb erstellt hatte.

Im Rahmen der Sozialhilfe gab es neben den Regelsätzen viele einmalige Beihilfen, mit denen individuell und zielgenau auf konkrete Bedarfslagen reagiert werden konnte. Problematisch war der hohe Verwaltungsaufwand, der der Zuweisung der Mittel zugrunde lag. Dies führte bei Trägern dazu, dass nicht alle notwendigen und zur Verfügung stehenden finanziellen Unterstützungsleistungen auch tatsächlich angeboten wurden und Betroffene häufig auf die berechtigte Inanspruchnahme verzichten mussten. Deshalb gab es schon länger Überlegungen, diese Beihilfen - ebenfalls gestützt durch statistische Erhebungen stärker zu pauschalieren und die Regelsätze um diese Pauschalen anzuheben. Dies wurde mit dem Übergang zum SGB II und SGB XII auch umgesetzt. Gleichzeitig wurde die Umstellung auf nunmehr nominal höhere Regelsätze zur verdeckten Kürzung gerade bei den ehemaligen einmaligen Beihilfen genutzt (DPWV 2004; Spindler 2004).

\subsection{UNTERKUNFTSKOSTEN}

Zu den bisher genannten Leistungen kommen noch Unterkunftskosten, einschließlich der Heizkosten. Hier ist die Regelung

\footnotetext{
Bemerkenswert war auch, dass für Haushaltsangehörige, vor allem für Kinder und Jugendliche, ei gene Warenkörbe gepackt wurden, die zu strukturell höheren Regelsätzen führten als heute: Statt $60 \%$ des Eckregelsatzes für Kinder bis 14 Jahre und $80 \%$ für Jugendliche, gab es $65 \%$ vom Eckregelsatz für Kinder ab 7 Jahre und $90 \%$ für Jugendliche ab 14 Jahre und das ursprünglich bis zum Alter von 21 Jahren.

2 Die umfangreiche Literatur zur Entwicklung der Regelsätze wird erschlossen in der Kommentierung von Roscher zu $\S 22$ BSHG und in den jeweiligen Auflagen des Lehr- und Praxiskommentars zum BSHG (zuletzt LPK- BSHG 2003). Hinzu kommen vor allem in der zweiten Hälfte der 1980er Jahre zahllose Aufsätze im Nachrichtendienst des deutschen Vereins, in den Blättern der Wohlfahrtspflege des Paritätischen Wohlfahrtsverbands (DPWV), in Theorie und Praxis der sozialen Arbeit der Arbeiterwohlfahrt (AWO) und in vielen sozialrechtlichen und sozialpolitischen Fachzeitschriften.
} 
aus der bisherigen Sozialhilfe übernommen worden. Die Unterkunftskosten werden entsprechend dem in Deutschland sehr unterschiedlichen Mietniveau (die Begleitstatistik zum Wohngeldgesetz unterscheidet bereits zwischen sechs Mietstufen) und der Größe der Haushalte zunächst in tatsächlicher Höhe und nach einem halben Jahr in (orts-)angemessener Höhe übernommen, falls ein Wohnungswechsel möglich und zumutbar ist (vgl. $\$ 22$ Abs. 1 SGB II).

Obwohl die Kostenträgerschaft im SGB II bei den Kommunen verblieben ist, hat die Implementierung des neuen Gesetzes keine Entlastung gebracht, sondern vielmehr zu einem zusätzlichen Spardruck zulasten der existenzsichernden Leistungen geführt. Dies lag erstens daran, dass die Kommunen nunmehr mit dem Mietkostenbedarf der ehemaligen Arbeitslosenhilfebezieher konfrontiert wurden. Gleichzeitig wurde ihnen der Wohngeldbetrag, den sie vorher im Gegenzug einnehmen konnten, gestrichen. Dazu kam zweitens, dass nicht nur die Fallzahlen, sondern auch die Mietnebenkosten, die ebenfalls übernommen werden müssen, stark anstiegen. Damit war die Entlastung, die ihnen ohne genaue Kalkulationsgrundlagen versprochen worden war, zunichte gemacht. ${ }^{3}$

\section{Zum Verhältnis zwischen sozialhilferechtlichem Existenzminimum und Löhnen}

In welcher Relation steht das für alle geltende Existenzminimum aus Regelsätzen/ Mehrbedarf und Unterkunftskosten zur Arbeitswelt und insbesondere zu den Löhnen?

Die Antwort lautet: in keiner. Denn typisch arbeitsbedingte Ausgaben und Bedarfe sind in dem sozialhilferechtlichen Existenzminimum nicht enthalten. Es geht bei den anerkannten Bedarfen um die menschlichen Grundbedürfnisse: Essen, Haushaltsführung und Kleidung, um das Dach über dem Kopf, um minimale Mobilität und Teilhabe am kulturellen Leben kurz: es ist das Existenzminimum der Nichterwerbstätigen. Deshalb ist auch die Aussage falsch, es gäbe zwar keinen offiziellen Mindestlohn in Deutschland, aber das oben beschriebene Niveau des Existenz- minimums sei praktisch der Mindestlohn. Durch das Teilhabegebot und verstärkt durch das Statistikmodell fließen zwar indirekte Bezüge zum Verbraucherverhalten von Niedrigeinkommensbeziehern ein, zu denen auch Niedriglohngruppen gehören. Gleichzeitig deutet einiges daraufhin, dass sich in dieser Vergleichsgruppe auch eine relativ hohe Zahl von Sozialleistungsbeziehern wie z. B. Rentnern findet. Dennoch haben Armutsgrenzen, wie etwa Anteile von $40 \%$, $50 \%$ oder mehr der Durchschnittslöhne, oder erkennbare Bezüge zu Mindestlöhnen, wie etwa in den Niederlanden, bisher bei der Festlegung der Leistungshöhe keine Rolle gespielt.

Die Sozialhilfeleistung hat in Deutschland auch nie die Funktion eines Mindestlohns übernommen. Im Gegenteil: Sie hat, wie wir aktuell feststellen können, keine Lohnabsenkungen unter die Schwelle des Existenzminimums aufhalten können. Gerade die durchgehend hohe, bis zu 50 \%ige Dunkelziffer speiste sich schon immer aus Erwerbstätigen mit zu niedrigem Lohn, die nichts über ergänzende Ansprüche wussten oder die von der Verwaltung oder dem Randgruppenimage der Sozialhilfe abgeschreckt waren. Die Löhne haben sich unabhängig von diesem Betrag nach unten entwickelt und glichen sich nachweislich nicht an.

Die Sozialhilfeschwelle wirkte allerdings als Referenzgröße für Lohnhöhen insbesondere für Niedriglöhne. Aber anstatt sich mit diesem Problem auseinander zu setzen, wurde dann rasch der Vorwurf der "Sozialstaatsfalle“ ins Gespräch gebracht: Nicht etwa, weil bestimmte Löhne zu niedrig, sondern weil das staatliche Existenzminimum zu hoch sei, würden sich die Leute nicht genug um niedrig entlohnte Arbeit bemühen. Dagegen spricht die hohe Dunkelziffer der working poor. Dagegen spricht auch die Tatsache, dass gerade Familienvorstände, bei denen das Lohnabstandsgebot am ehesten verletzt wird, die größte Gruppe derer stellen, die niedrige Einkommen mit ergänzenden Leistungen kombinieren und nicht etwa das Arbeiten aufgeben (Bofinger et al. 2006, S. 37). AuBerdem ist dem entgegenzuhalten, dass wegen des noch darzustellenden Bedarfs in der Sozialhilfe für Erwerbstätige jeder Hilfebezieher bei Aufnahme einer Arbeit im Ergebnis etwas mehr Geld zur Verfügung haben muss als zuvor.

Es ist ein spezifisch deutsches Problem, dass der Staat zwar existenzsichernde Leis- tungen im Rahmen der Fürsorge bzw. der Sozialhilfe gewährleistet hat, gleichzeitig aber nicht auf die existenzsichernde Höhe der Löhne geachtet hat. So gibt es zwar eine Tradition, Fürsorgeleistungen für Nichterwerbstätige, Leibrenten oder Mieten an den Lebenshaltungskostenindex zu binden, nicht aber die Löhne am unteren Rand. Im Gegenteil: Als sich nach langer Zeit des wirtschaftlichen Aufschwungs, in der die Tarifpartner für ein steigendes Lohnniveau unterer Lohngruppen gesorgt hatten, das Problem nicht existenzsichernder Löhne langsam ankündigte, kam fast unbemerkt in den 1990er Jahren ein merkwürdig formuliertes Lohnabstandsgebot mit $\$ 22$ Abs. 4 BSHG in das Gesetz. Als Vergleichsgröße für die Ermittlung der Regelsätze sollte das nicht näher definierte durchschnittliche Nettoarbeitsentgelt unterer Lohngruppen eines Alleinverdieners mit einem fünfköpfigen Haushalt zuzüglich Kinder- und Wohngeld herangezogen werden. ${ }^{4}$ Diese Lohnabstandsformel hat bis heute zwar wenig Schaden angerichtet. Ihr Problem liegt vielmehr darin, dass sich ein Lohnabstand nur dann sinnvoll bestimmen lässt, wenn ein Mindestlohn festgelegt wird.

Leider bieten auch die Regeln des Bürgerlichen Gesetzbuches (BGB) und die Arbeitsgerichtsrechtsprechung zur Sittenwidrigkeit von Wucherlöhnen keinerlei Garantie dafür, dass Niedriglöhne zur Existenzsicherung ausreichen. Zwar sehen einzelne Arbeitsgerichte vor allem in einem dem Arbeitslosen aufgezwungenen Lohn, der nicht existenzsichernd ist, einen Tatbestand der Sittenwidrigkeit (Spindler 2003, S. 58). Dies wurde inzwischen auch in Entscheidungen über die Zumutbarkeit der Ar-

\footnotetext{
3 Im Prinzip wäre eine Pauschalierung einschließlich der Unterkunftskosten möglich. Aber die Frage, wie die regionalen Unterschiede auszugleichen und preiswerte Formen des Zusammenlebens in Wohngemeinschaften zu bewerten wären, müsste gelöst werden. Alle bisherigen Pauschalierungsexperimente ( $z$. B. Kassel) haben die Pauschale zu niedrig angesetzt und Folgeprobleme bei der Wohnraumversorgung ausgelöst.

4 Das Nettoarbeitsentgelt eines Alleinverdieners in einem 5-Personenhaushalt wäre dann sinnvoll, wenn die meisten Wirtschaftszweige in den unteren Lohngruppen ohne Schwierigkeiten Löhne aufbringen könnten, von denen man eine solche Familie ernähren kann. Dazu ist die Wirtschaft aber nicht in der Lage, und es ist auch nicht nötig, weil nur ein geringer Anteil aller Haushalte (ca. 2$3 \%$ ) so groß ist, und ein noch kleinerer Teil davon hat nur einen Hauptverdiener und den auch noch in einer unteren Lohngruppe.
} 
beitsaufnahme von mehreren Sozialgerichten übernommen. Die Rechtsprechung des Bundesarbeitsgerichts steht aber dieser Auffassung bisher ablehnend gegenüber. ${ }^{5}$

\section{4 \\ Das Existenzminimum von Erwerbstätigen}

Das staatlich gesicherte Existenzminimum hat noch ein weiteres Bedarfselement, das häufig nicht wahrgenommen wird: den geschützten Bedarf des Erwerbstätigen. Das war im BSHG bis 1993 deutlich an dem Mehrbedarf für Erwerbstätige ( $\$ 23$ Abs. 5 BSHG) zu erkennen. Dieser Mehrbedarf für Erwerbstätige, der zwischen $25 \%$ und $66 \%$ eines Regelsatzes lag, wurde durch den Deutschen Verein schon frühzeitig mit einer Berechnungsformel, bestehend aus Grundfreibetrag und Steigerungsbetrag, gefüllt (Brühl, LPK BSHG, 6. Aufl. 2003, $\$ 76$ Rnr. 104 ff.). Damit hatte man immer noch keinen Mindestlohn, sondern eine individuelle, teilweise familienbezogene, fürsorgerechtliche Existenzsicherung bei Niedriglöhnen.

Der Mehrbedarf hatte zwei Funktionen: Er sollte einen sogenannten unbezifferbaren Mehrbedarf für Erwerbstätige sichern (z. B. erhöhte Aufwendungen für Kleidung und Körperpflege, Bildungsaufwand, gesellige Verpflichtungen am Arbeitsplatz, verteuerter Einkauf aus Zeitknappheit, gelegentliches Auswärtsessen und Erholungsbedarf) und eine kleine Anreizfunktion erfüllen, die ich eher als Anteil zur Verbesserung der Lebensqualität bezeichnen würde (Spindler 2000). Zusätzlich konnten Erwerbstätige aber auch noch bezifferbare Werbungskosten wie Fahrtkosten, Arbeitsmittel, Beiträge zu Berufsverbänden und Versicherungen, z. B. auch zur Riesterente, vom anrechenbaren Einkommen absetzen. 1993 verschob man den Mehrbedarf in gleicher Höhe in den Status eines Einkommensfreibetrags. Das geschah, um nach der Entscheidung des Bundesverfassungsgerichts das steuerfreie Existenzminimum niedrig zu halten, das ja sonst um diesen Mehrbedarf hätte erhöht werden müssen (Brühl, LPK BSHG, 6. Aufl. 2003, $\$ 76$ Rnr. 80 ff.). Bis heute ist im Steuerrecht deshalb nur das Existenzminimum des nicht Erwerbstätigen steuerfrei. In den sogenannten Existenzminimumsberichten wird, anders als die Überschrift suggeriert, nicht etwa das gesamte Existenzminimum ermittelt, sondern es werden die in der Sozialhilfe gesetzten Regelleistungen für Nichterwerbstätige übernommen, und für Mieten wird ein (z. T. mit nicht genau bezeichneten Wohngeldbeträgen gerechtfertigter) sehr niedriger Pauschbetrag eingesetzt, der bisher nie inhaltlich auf seine Höhe überprüft wurde. ${ }^{6}$ Seither wurde aber nicht nur im Steuerrecht, sondern auch im Fürsorgerecht an diesem Betrag manipuliert. Es dauerte nicht lange, bis Sozialhilfeträger diesen Betrag, der seines Mehrbedarfscharakters durch die Umstellung entkleidet worden war, absenkten. Seine Bedeutung wurde dabei unmerklich verändert: Es ging angeblich nicht mehr um einen Mehrbedarf, sondern nur noch um einen Anreiz zur Arbeitsaufnahme, den man nunmehr auf Unmotivierte konzentrieren wollte, denn die Arbeitswilligen brauchten bei diesem Verständnis ja keinen Anreiz mehr (Spindler 2000; 2002).

Im neuen SGB II sind die bezifferbaren Werbungskosten und Versicherungsbeiträge seit dem 1.10.2005 mit einem Grundbetrag von $100 €$ in $\$ 11$ Abs. 2 SGB II abgegolten. Dies mag im Einzelfall nicht überzeugend sein und kann in bestimmten Fällen erhöht werden. Im Ergebnis stellt dieser Grundbetrag jedoch für die meisten Vollzeitarbeitenden eine noch angemessene Pauschale dar. Der weitere Freibetrag für Erwerbstätigkeit wird in einem Steigerungsbetrag von zunächst $20 \%$ und später $10 \%$ des Brutto-Resteinkommens in $\$ 30$ SGB II geregelt. Dies entspricht in der Struktur und auch in der Höhe den ursprünglichen Empfehlungen des Deutschen Vereins zum Mehrbedarf für Erwerbstätige. Auch wenn insbesondere der Freibetrag für Erwerbstätige immer nur ein abgeleiteter Prozentsatz ist und nicht mit einem Warenkorb oder tatsächlichen Daten unterlegt war, so wäre er heute eher höher anzusetzen als früher in der Sozialhilfe. Nicht nur der unbezifferbare Mehrbedarf ist angewachsen, auch viele Vergünstigungen, die früher allen Niedrigeinkommensbeziehern und nicht nur Sozialhilfeempfängern zugute gekommen waren, sind weggefallen. Das gilt z. B. für ergänzende einmalige Beihilfen und Krankenhilfe, neuerdings für die Befreiung von der GEZ-Gebühr oder für viele kommunale Vergünstigungen. Allein die Belastungsgrenze bei den Gesundheitsausgaben ist nicht wie bei Arbeitslosengeld II-Beziehern auf $82 €$ pro Jahr begrenzt, sondern wegen des Bruttolohnbezugs auf den gesamten Niedriglohn etwa dreimal so hoch.

Diese erwerbsbedingten Frei- und Absetzbeträge sind nicht $\mathrm{zu}$ verwechseln mit den vielen Kombilohnmodellen, die immer befristet konzipiert waren und nur bereits arbeitslos Gemeldete wieder aus der Arbeitslosigkeit hinausführen sollten. Freiund Absetzbeträge gelten für alle Niedrigeinkommensbezieher, d.h. auch für Selbstständige und Beamte und für all jene, die vorher keine Leistungen wegen Arbeitslosigkeit bezogen haben. Sie sind damit egalitär auf die Absicherung eines Mindestbedarfs bzw. des Existenzminimums für alle Erwerbstätigen ausgerichtet und stellen eine existenzsichernde, individuelle Lohnaufstockung dar, die umso höher ausfällt, je niedriger die Löhne sind.

Der finanzielle staatliche Aufwand kann nur in Grenzen gehalten werden, wenn auf der anderen Seite Mindestlöhne eingezogen werden, die dann nur noch bei bestimmten Bedarfssituationen (z. B. größeren Haushalten, Teilzeitarbeit) ergänzt werden müssen oder zu bestimmten Zwecken (z. B. Ausbildungs- oder Anlernverhältnisse) begründet unterschritten werden dürfen.

Ansonsten macht ein verlässlicher Mehrbedarf bzw. heute: Frei- und Absetzbetrag alle weiteren Kombilohnexperimente überflüssig. Er stellt einen positiv formulierten Lohnabstand her und kann kleine Unstimmigkeiten in den übrigen Bedarfsfestsetzungen ausgleichen.

\section{Bestimmung eines existenzsichernden Mindestlohns}

\subsection{EIGENE BERECHNUNGEN}

Bei einer Gesamtbetrachtung ergibt sich, dass viele Elemente des deutschen Exis-

\footnotetext{
5 Vgl. dazu die BAG-Entscheidung vom 23.4.2004 - 5 AZR 303/03, mit der für die deutsche Entwicklung typischen Begründung, dass das Sozialhilfeniveau von anderen Faktoren abhängig sei als der Arbeitsleistung. Art. 4 der Europäischen Sozialcharta $_{\perp}$ aus der man ein Recht auf existenzsichernde Löhne ableiten könne, sei insoweit rechtlich unverbindlich.

6 Vgl. dazu den Sechsten Existenzminimumsbericht vom 2.11.2006, BT - Drucksache 16/ 3265 vom 2.11. 2006.
} 
tenzminimums eine enge Beziehung zu einem für eine einfache Existenz notwendigen Lebensunterhalt haben und damit eine Indexbindung an die Lebenshaltungskosten bewirken. Und weil es in Deutschland bisher keine vergleichbaren Untersuchungen und Initiativen gibt, derartiges für die Lohnfindung am unteren Rand zusammenzustellen, wird hier vorgeschlagen, Elemente aus dem sozialhilferechtlichen Existenzminimum für die Bestimmung eines Mindestlohnes heranzuziehen. Denn auch ein Niedriglohnbezieher muss in dieser Gesellschaft existieren können.

Im Rahmen einer Untersuchung über einen zumutbaren existenzsichernden Mindestlohn habe ich deshalb Anfang 2003, noch unter Geltung des BSHG, zusammengestellt, wie hoch dieser Lohn für eine alleinstehende Person hätte ausfallen müssen. In Tabelle 1 füge ich außerdem hinzu, wie es 2006 mit den entsprechenden Beträgen des SGB II bzw. unter Berücksichtigung des alternativen Regelsatzvorschlags des DPWV aussehen müsste, wenn man einen wirklich existenzsichernden Lohn bestimmen will.

Meine Berechnung für das Jahr 2003 habe ich an anderer Stelle genauer begründet (Spindler 2003, S. 59 f.). Regelsätze und einmalige Beihilfen entsprechen den staatlichen Festsetzungen bzw. statistischen Erfahrungswerten. Auch der Regelsatz des DPWV stützt sich auf statistische Erhebungen. Bei der Miete konnte ich mich im Jahr 2003 noch auf die relativ zeitnahen Erhebungen zum Wohngeldgesetz stützen. Über inzwischen gestiegene Neben- und Heizkosten liegen mir keine genaueren $\mathrm{Da}$ ten vor. Deshalb habe ich zunächst die Beträge als konstant unterstellt.

Die Mehrbedarfe, die ganz stark mit persönlichen Lebensumständen zu tun haben, gehen in die Überlegungen nicht mit ein und müssten weiterhin durch öffentliche Zuwendungen kompensiert werden. Wohl aber müssen in die Lohnfindung die bezifferbaren Werbungskosten und unbezifferbaren Frei- und Absetzbeträge einfließen, die ja mit einem Lohn erst einmal erwirtschaftet und auch sofort wieder verbraucht werden müssen. Für die Sozialhilfe habe ich die bezifferbaren Werbungskosten und Versicherungen mit $30 \%$ geschätzt. Im SGB II ist die gesetzliche Pauschale von $100 €$ eingesetzt, die man beispielsweise mit $60 €$ Fahrtkosten, einem Gewerkschaftsbeitrag und Beiträgen zur Riesterrente und einer Haftpflichtversiche-

\begin{tabular}{|c|c|c|c|}
\hline & Anfang 2003 BSHG & 2006 SGB XII/II & 2006/DPWV \\
\hline $\begin{array}{l}\text { Bedarfstatbestand } \\
\text { Regelleistung/Eckregelsatz }\end{array}$ & $293,00 €$ & $345,00 €$ & $415,00 €$ \\
\hline $\begin{array}{l}\text { Einmalige Beihilfen } \\
20 \% \text { davon im BSHG, } \\
\text { später im Regelsatz enthalten }\end{array}$ & $58,00 €$ & - & - \\
\hline $\begin{array}{l}\text { Miete ( } 40 \text { qm } 5,70 € / q m \\
\text { zzgl. } 1,30 € / q m \text { kalte Nebenkosten) } \\
\text { Heizung }(1,00 € / q m)\end{array}$ & $\begin{array}{r}280,00 € \\
40,00 €\end{array}$ & $\begin{array}{r}280,00 € \\
40,00 €\end{array}$ & $\begin{array}{r}280,00 € \\
40,00 €\end{array}$ \\
\hline $\begin{array}{l}\text { Alle bezifferbaren Absetzbeträge } \\
\text { BSHG § } 76 \text { Abs. } 2 \\
30 \% \text { vom Regelsatz pauschal } \\
\S 11 \text { Absatz } 2 \text { SGB II pauschal }\end{array}$ & $88,00 €$ & $10 \overline{0}, 00 €$ & $100,00 €$ \\
\hline $\begin{array}{l}\text { Erwerbstätigenfreibetrag } \\
\text { BSHG § } 76 \text { Abs. } 2 \mathrm{a} \\
50 \% \text { vom Regelsatz } \\
\S 30 \text { SGB II, } 20 \% \text { von } 700,00 € \\
\text { plus } 10 \% \text { vom weiteren Einkommen }\end{array}$ & $\begin{array}{c}146,00 € \\
-\end{array}$ & $180,00 €$ & $180,00 €$ \\
\hline $\begin{array}{l}\text { Notwendiges Nettomonatseinkommen } \\
\text { für eine erwerbstätige Person }\end{array}$ & $905,00 €$ & $945,00 €$ & $1.015,00 €$ \\
\hline $\begin{array}{l}\text { Das entspricht einem } \\
\text { Nettostundenlohn bei } \\
173 \text { Std. (= } 40 \text { Std./Woche) } \\
164 \text { Std. (= } 38 \text { Std./Woche) }\end{array}$ & $\begin{array}{l}5,23 € \\
5,52 €\end{array}$ & $\begin{array}{l}5,46 € \\
5,77 €\end{array}$ & $\begin{array}{l}5,87 € \\
6,19 €\end{array}$ \\
\hline
\end{tabular}

rung schon ausgefüllt haben dürfte. Schließlich ist der Freibetrag im BSHG nach den Empfehlungen des Deutschen Vereins eingesetzt und im SGB II nach der Vorschrift des $\$ 30$ SGB II, was in diesem Einkommensbereich zu vergleichbaren Ergebnissen führt. Die Regelleistung, die der DPWV ermittelt hat, entspricht den tatsächlichen Lebenshaltungskosten und aktuellen Preissteigerungen eher als der vielfach kritisierte Regelsatz von $345 €$, der viele unbegründete Abstriche am notwendigen Bedarf vornimmt. Ein für Deutschland fairer Lohn müsste deshalb eher bei $1.015 €$ im Monat liegen. „Fair“ ist hier in einem Sinn gebraucht wie im amerikanischen Fair Labour Standard Act (FLSA). Auch wenn dieses Gesetz in den USA nicht automatisch faire Löhne garantiert, steht es doch für den gesellschaftlichen Anspruch, einen Lohn festzusetzen, von dem man leben kann, so wie das 2007 nach langen Verzögerungen erneut durch Beschluss des US-Senats versucht wird.

Obwohl die gegenwärtige Diskussion mehr von Nettoeinkommensgrenzen bestimmt ist, was mir beim Vergleich mit dem sozialrechtlichen Existenzminimum entgegenkommt, möchte ich auch die Auswirkungen auf das Bruttoeinkommen für 2006 andeuten (Tabelle 2). Ich berücksichtige dabei jeweils $21 \%$ Arbeitnehmeranteil zur Sozialversicherung und ein Gesamtsteueraufkommen aus Lohn- und Kirchensteuer und Solidaritätszuschlag von $74,39 €$, bzw. für die DPWV-Variante von $123,44 €$.

\subsection{ALTERNATIVE MODELLE}

Die in den Tabellen 1 und 2 dargestellten Ergebnisse weichen von anderen Vorschlägen ab, die nun kurz vergleichend betrachtet werden sollen:

Vier der Wirtschaftsweisen wollen in ihrer Empfehlung den bestehenden Regelsatz für Nicht-Erwerbstätige um ein Drittel kürzen und nur dann voll auszahlen, wenn dafür gemeinnützig gearbeitet wird. Sie schlagen vor, die gegenwärtig ca. 300000 Mehraufwandsbeschäftigungen (1-EuroJobs) mehr als zu verdoppeln. Ohne die Kritik am Missbrauch dieses sozialrechtlichen Beschäftigungsinstruments hier ver-

\begin{tabular}{|c|c|c|}
\hline Bruttomonatslohn 2006 & $1.290,00 €$ & $1.440,00 €$ \\
\hline $\begin{array}{l}\text { Bruttostundenlohn bei } \\
173 \text { Std. (=40 Std./Woche) } \\
164 \text { Std. (= } 38 \text { Std./Woche) }\end{array}$ & $\begin{array}{l}7,46 € \\
7,86 €\end{array}$ & $\begin{array}{l}8,32 € \\
8,78 €\end{array}$ \\
\hline
\end{tabular}


tiefen zu können, basiert dieser Vorschlag auf zwei Fehlschlüssen bezüglich des Existenzminimums: Erstens stellt die Regelleistung - wie bereits oben erwähnt - das Minimum des Nichterwerbstätigen dar. Zweitens erhöht sich, wenn er in dem gewünschten Umfang beschäftigt wird, sein Existenzbedarf über die Regelleistung hinaus, denn die Arbeit bedingt einen bezifferbaren und unbezifferbaren Mehraufwand, der nicht im Regelsatz enthalten ist. Deswegen gab es früher im BSHG und gibt es heute im SGB II auch die sogenannte angemessene Mehraufwandsentschädigung von 1,00 bis $1,50 €$ pro Stunde.

Andere Vorschläge macht eine Arbeitsgruppe um Bofinger und Walwei (Bofinger et al. 2006). Sie nimmt von der Forderung nach Senkung der Regelleistung für Nichterwerbstätige und der damit verbundenen Ausweitung der Arbeitsgelegenheiten mit Mehraufwandsentschädigung Abstand und plädiert im Rahmen eines umfassenden Maßnahmenbündels für eine Abgabensenkung im Niedriglohnbereich durch Einkommensteuergutschrift (Bofinger et al. S. 89f.). Dies wird verbunden mit einem abgabefreien Mindesteinkommen bei Vollzeitbeschäftigung von $750 €$ netto für Alleinstehende und entspricht einem Stundenlohn von $4,50 €$ bei einer Wochenarbeitszeit von 38 Stunden (ebd., S. 105). Dieser Vorschlag zielt zunächst, durch die Einbindung in den arbeits- und sozialversicherungsrechtlichen Schutz, auf den regulären Arbeitsmarkt, ohne das Existenzminimum für Nichterwerbstätige zu beschädigen.

Die ins Auge gefasste Lohnhöhe liegt allerdings sehr deutlich unter der, die oben entwickelt wurde. Dies ist erstaunlich, da die Autoren selbst mit Bezug auf international vergleichende Daten auf Mindestlöhne zwischen 7,00 und 8,69€ brutto (beginnend von den Neuengland-Staaten in den USA bis hin nach Luxemburg) verweisen (ebd., S. 32). Eine Erklärung für diesen im Vergleich niedrig angesetzten Mindestlohn liegt darin, dass er, entsprechend der bereits angesprochenen deutschen Tradition, aus verschiedenen Überlegungen und Relationen, aber nicht aus den tatsächlich bestehenden Bedarfen und Lebenshaltungskosten abgeleitet wird. Die Autoren versuchen zwar, ihn aus der Summe von aktuellem Regelsatz, pauschalierten Unterkunftskosten und einem Zuschlag zu ermitteln, setzen die Bedarfe aber, selbst verglichen mit der gegenwärtigen Rechtslage, zu niedrig an.
Selbst wenn man darüber hinwegsieht, dass der Regelsatz von $345 €$ unkritisch zugrunde gelegt wird, sind vor allem die weiteren Annahmen unter Aspekten der Existenzsicherung nicht vertretbar. So unterstellen die Autoren einen notwendigen durchschnittlichen Unterkunftsbedarf von $219 €$ (ebd., S. 36). Der statistische Durchschnittswert, der hier ohne nähere Begründung eingeführt wird, ist für eine Mindestlohnbestimmung ungeeignet. Anzunehmen, dass davon in Deutschland eine ca. $40 \mathrm{qm}$ Wohnung (warm) für einen alleinstehenden Erwerbstätigen anzumieten sei, ist lebensfremd; für diesen Betrag ist kaum mehr ein (subventioniertes) Studentenzimmer zu bekommen. Selbst die eher fiktive Annahme von Wohngeld macht die Kalkulation nicht ausreichend.

Die in der Studie dann noch verbleibende Differenz zwischen 564 und $750 €$, nämlich $186 €$, berücksichtigt zwar noch einen zusätzlichen Bedarf für Erwerbstätige, liegt aber sehr deutlich unter den $280 €$ an Freibetrag und Werbungskostenpauschale, die gegenwärtig im Sozialrecht begründet anerkannt werden. Nimmt man eine angemessene Miete an, dann bleiben von den $750 €$ sogar nur noch ca. $86 €$ für diesen Zweck übrig. Die Autoren argumentieren zwar, dass Freibeträge für die Minijobs niedriger werden müssten, aber warum sie auch beim Vollzeitbeschäftigten so deutlich sinken sollen, wird nicht erläutert. Die vorgeschlagene Mindestlohnhöhe von $750 €$ kommt so durch empfindliche Einschnitte im Mietniveau und bei den berücksichtigten Werbungskosten/Freibeträgen zustande, die den tatsächlichen Bedarf und die Belastungen der Niedrigeinkommensbezieher genauso wenig berücksichtigen wie die bisherigen Anrechnungspraktiken und ihre Begründung in den Fürsorgesystemen.

\section{Negative Einkommen- steuer statt aktueller Bedarfsdeckung?}

Schließlich verlässt auch die verfahrenstechnische Vorstellung, Niedriglohnbezieher zunächst zu Abgaben heranzuziehen und ihnen erst im Nachhinein eine bedarfsorientierte Steuergutschrift zu erstatten (negative Einkommensteuer), die bisherige Verfahrenspraxis der Existenzsiche- rung. Wer am Existenzminimum lebt, ist auf aktuelle Bedarfsdeckung - sei es durch Nettolohn oder durch ergänzende Sozialleistungen - angewiesen und kann nicht beliebig vorleisten und auf Rückerstattungen warten. Die Ermittlung eines existenzsichernden Freibetrags durch eine nicht auf soziale Problemlagen ausgerichtete Steuerbehörde wäre zudem etwas anderes als der vergleichbar problemlose Eintrag eines Steuerfreibetrags, der ohne Bedarfsbezug nur aktuell mehr vom zufließenden Einkommen belässt. Die bei existenzsichernden Leistungen nie ganz zu vermeidende Bedürftigkeitsprüfung käme hinzu. Auch besteht das Problem, dass zu Beginn des Jahres die möglichen steigenden Einkommen im weiteren Verlauf noch nicht geschätzt werden können und die Betroffenen deshalb zu vertrösten wären. Die negative Einkommensteuer kann im Übrigen nicht wirken, wenn, wie in den obigen Beispielen dargestellt, bereits auf knapp existenzsichernde Löhne schon eine „positive" Einkommensteuer erhoben wird. In Deutschland müsste daher zunächst einmal das steuerfreie Existenzminimum auf eine entsprechende Höhe angehoben werden, was schon eine Entlastung der Lohnkosten bewirken würde, ohne das Nettoeinkommen zu schmälern. Wenn man darüber hinaus staatliche Unterstützung vorsehen will, würde jeder nur auf die Erhöhung des Nettolohns des Arbeitnehmers ausgerichtete Zuschuss, wie die negative Einkommensteuer, die bruttobezogenen Sozialversicherungsabgaben auf zu niedrigem Niveau belassen, was erhebliche Folgen für diese Systeme selbst und ihre existenzsichernde Funktion bei Eintritt des Versicherungsfalles hat. Auch Lohnersatzleistungen wie Arbeitslosengeld oder Rentenanwartschaften erreichen nur eine annähernd existenzsichernde Höhe, wenn sie von einer Lohnhöhe wie hier ermittelt berechnet werden. Ein Zuschuss, der diese Wirkungen vermeiden und Arbeitgeber entlasten will, die nicht in der Lage sind, diesen Mindestlohn zu erwirtschaften, müsste deshalb auf den Sozialversicherungsanteil der Arbeitgeber zielen. Doch dafür ist die negative Einkommensteuer ebenfalls ungeeignet; besser wäre, wie in den USA angedacht, eine Steuergutschrift für Unternehmer.

Die negative Einkommensteuer ist eine in Deutschland systemfremde Steuer. Sie birgt deshalb erhebliche Risiken und Nebenwirkungen, die man mit einem bisher 
auch unüblichen, aber nach den Vorarbeiten im Fürsorgerecht durchaus bestimmbaren Mindestlohn vermeiden würde. Anschließend könnte dann eine verlässliche Indexbindung dieses Lohns in Angriff genommen werden, für die wesentlich mehr öffentliche Aufmerksamkeit zu mobilisieren wäre als für die Erhebung zur Festsetzung der Sozialhilferegelsätze, deren Bedeutung von vielen unterschätzt wird. Und darauf könnte man ein Konzept aufsetzen, wie Familien mit Kindern in ihrer
Existenz gesichert werden - denn auch das ist ohne einen verlässlichen Ausgangspunkt schwer möglich, wie zuletzt der untaugliche Versuch der Einführung des Kinderzuschlags deutlich gemacht hat.

\section{LITERATUR}

Bofinger, P./Dietz, M./Genders, S./Walwei, U. (2006): Vorrang für das reguläre Arbeitsverhältnis: Ein Konzept für Existenz sichernde Beschäftigung im Niedriglohnbereich. Gutachten für das Sächsische Ministerium für Wirtschaft und Arbeit, August 2006

Bundessozialhilfegesetz (2003): Lehr- und Praxiskommentar, LPK-BSHG, 6. Aufl., Baden-Baden

Der Paritätische Wohlfahrtsverband (DPWV) (Hrsg.) (2004): Expertise Zum Leben zu wenig ... Für eine offene Diskussion über das Existenzminimum beim Arbeitslosengeld II und in der Sozialhilfe, Berlin Der Paritätische Wohlfahrtsverband (DPWV) (Hrsg.) (2006): Neue Regelsatzberechnung 2006, Berlin

Leibfried, S. (1976): Armutspotential und Sozialhilfe in der Bundesrepublik. Zum Prozess des Filterns von Ansprüchen in der Sozialhilfe, in: Kritische Justiz 9, S. 376-393
Martens, R. (2006 ): Neue Regelsatzberechnung 2006. Zu den Vorschlägen des paritätischen Wohlfahrtsverbandes und der Bundesregierung, in: Soziale Sicherheit 6, S. 182-194

Schulte, B./Trenk-Hinterberger, P. (1986): Sozialhilfe. Eine Einführung, Heidelberg

Spindler, H. (2000): Der Erwerbstätigenfreibetrag - seine Elemente und seine sozialpolitische Funktion, in: info also 4, S. 183-186

Spindler, H. (2002): Anmerkung zur Entscheidung des Bundesverwaltungsgerichts vom 21.12., in: info also 4, S. 180-181

Spindler, H. (2003): Grenzen der Zumutbarkeit von Arbeit für Sozialhilfeberechtigte bei Niedriglöhnen und Lohnwucher, in: info also 2, S. 56-62 Spindler, H. (2004): Die neue Regelsatzverordnung - Das Existenzminimum stirbt in Prozentschritten, in: info 4, S. 147-151 